\title{
Technology for aetiology-based diagnostics
}

\author{
Accurate diagnostics need technology - from imaging hardware and image reconstruction to machine learning - \\ to detect markers associated with the cause of disease.
}

$\mathrm{D}$ iagnosis involves the identification of abnormal characteristics associated with pathological conditions. As part of a decision-making process, diagnostic approaches assume that diseases can be readily classified by the extent to which a chosen set of parameters deviates from its healthy norm. The severity of a condition is then often assessed by the magnitude of such deviation. Traditionally, a diagnosis is performed by physicians, who assign, for each disease, a list of observable symptomatic characteristics that need to be informative of pathology and guide decision-making. The more precise the diagnosis, the more effective the treatment planning and overall prognosis. But reaching the wrong diagnosis can dramatically change how a patient is assessed and treated. Both accuracy and precision in diagnostic assessment are therefore essential to reach a high level of certainty about the choice of treatment (when available). Over time, advances in diagnostic tests can help refine disease classification (in particular when the information gathered involves markers of the origin of disease rather than its symptoms). For example, cancers used to be exclusively defined by their tissue of origin, yet progress in the molecular classification of disease subtypes has helped improve patient classification (for instance, cancer staging can now be linked to molecular drivers and biomarkers of cancer), refine treatment planning (because therapies can be targeted to activate or inhibit a specific molecular pathway), and enable the development and the assessment of anticancer drugs.

In today's landscape of data-driven molecular information, diagnostic approaches are less about a physician's set of observations and more about enhanced accuracy and precision in disease classification that leverages the understanding of disease mechanisms. This ability to integrate disease aetiology in diagnostics represents an exciting step forward: where symptomatic observations were correlated to a pathological condition but unlinked to the disease drivers, treatment planning largely relied on the physician's experience. Modern diagnostic devices, methods and tests, on which precision medicine is flourishing, can help the physician pair the status of the

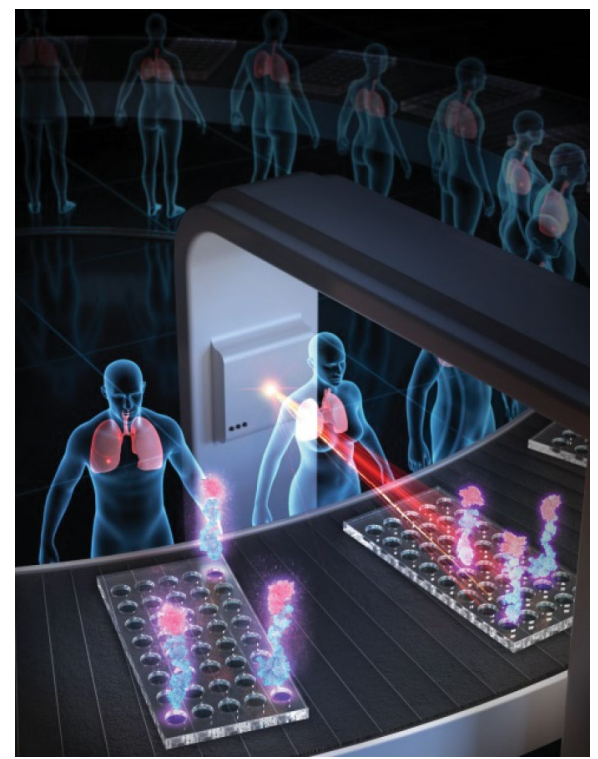

Credit: Young Jae Kim, 3Dgraphic, South Korea

disease with a drug that targets its causes (rather than its symptoms) with increased sensitivity and specificity.

The technological solutions for diagnostics included in this issue of Nature Biomedical Engineering embody these principles. Daniel Irimia and colleagues describe a fast microfluidic assay to accurately diagnose patients suffering from sepsis using a drop of their blood. The nature of this inflammatory condition can be so severe that speed and certainty in its diagnosis is critical in avoiding multiple organ failure. Indeed, patients at high risk for sepsis often receive antibiotics before the diagnosis is available (typically, from microbial-culture methods that take a few days), but the similarity in symptoms and biomarkers between a septic infection and inflammatory yet non-infectious conditions still leads to high rates of misdiagnosis. By training a machine-learning algorithm to analyse the complex motility patterns of neutrophils (the most abundant type of white blood cell) in a microfluidic device, the algorithm identified patients suffering from sepsis with a specificity of $98 \%$.

Matching patients to specific treatments is central in cancer. Tae-Young Yoon and colleagues show that responses to a targeted treatment for lung cancer are highly dependent on the formation of specific molecular signalling complexes. In cancer subtypes driven by a mutant epidermalgrowth-factor receptor, it is common to measure the level of phosphorylation of tyrosine residues as an indicator of signalling-pathway activity; yet the researchers found that the cumulative pathway activity is largely associated with the formation of multiple protein complexes, rather than with specific phosphorylation levels. By using single-molecule technology to profile protein-protein interactions that lead to the formation of these complexes (pictured), they identified lung-cancer subtypes that respond to therapies targeted to an inhibitor of the growth factor.

Because of accessibility, diseases of the skin should be detectable non-invasively; yet due to the anatomical similarities of skin conditions arising from the abnormal scarring of the skin after injury, biopsied tissue is typically required for accurate diagnosis. Chenjie Xu and collaborators demonstrate that abnormal fibrotic levels in the skin (such as those in hypertrophic scars and keloids) can be diagnosed by monitoring the expression of the connective-tissuegrowth-factor gene through topically applied nanotechnology developed to detect via fluorescence the levels of its specific mRNA. The technology is sensitive enough to monitor the efficacy of anti-scarring drugs.

Molecular and cellular information improves disease classification, yet the first port of call in diagnostic tests often relies on physiological readings, with radiological modalities such as X-ray computed tomography, ultrasound and magnetic resonance imaging (MRI) being routinely used to identify or rule out pathological conditions across the body. To observe the heart with MRI, however, image-acquisition protocols involve breath holds or echocardiography measurements as well as post-acquisition data sampling and filtering to decouple body movement (due to respiration and heart beating) from the dynamics of magnetic relaxation (that is, the measurements in MRI). By developing an image-reconstruction algorithm that can separate overlapping dynamics in the beating heart, Debiao Li and co-workers achieved unprecedented speed and quality in cardiovascular MRI, enabling the capture of previously unattainable information on heart dynamics. 
Because tissue significantly attenuates light, a strong signal from a deep-seated target and a weaker signal from a superficial site can be virtually indistinguishable. Hence, most optical imaging is in practice limited to depths of a few millimetres. Brian Pogue and colleagues show that X-ray beam-shaping from a linear accelerator can generate Cherenkov radiation (ultraviolet light emitted when charged particles travel through a dielectric medium faster than the speed of light in it) in vivo that can be unambiguously located at depths of tens of millimetres without loss in signal intensity. The technique was used to excite an oxygen-sensitive molecular probe to quantitatively map physiological oxygen pressure in human tumours implanted in mice, at submillimetre resolution and nanomolar sensitivity.
As the discovery and understanding of molecular markers linked to disease aetiology broadens, technological developments for their detection will further benefit clinical diagnostics.

Published online: 13 April 2018

https://doi.org/10.1038/s41551-018-0232-Z 